Acta Crystallographica Section E

Structure Reports

Online

ISSN 1600-5368

\title{
Pyrazine-2-carbohydrazide: a three-dimensional hydrogen-bonded framework structure
}

Molecules of the title compound, $\mathrm{C}_{5} \mathrm{H}_{6} \mathrm{~N}_{4} \mathrm{O}$, are linked into a three-dimensional framework structure by a combination of $\mathrm{N}-\mathrm{H} \cdots \mathrm{O}, \mathrm{N}-\mathrm{H} \cdots \mathrm{N}$ and $\mathrm{C}-\mathrm{H} \cdots \mathrm{O}$ hydrogen bonds.

\section{Comment}

As part of our general study of the supramolecular structures of amine and hydrazine derivatives, we report here the molecular and supramolecular structure of the title compound, (I). Within the hydrazino fragment, the coordination at $\mathrm{C} 7$ and $\mathrm{N} 2$ is planar within experimental uncertainty, while the coordination at N3 is markedly pyramidal (Fig. 1). Apart from the $\mathrm{H}$ atoms bonded to atom N3, the molecule is effectively planar, as shown by the key torsion angles (Table 1); the bond distances and angles show no unexpected features.

Correspondence e-mail: cg@st-andrews.ac.uk

\author{
Key indicators \\ Single-crystal X-ray study \\ $T=120 \mathrm{~K}$ \\ Mean $\sigma(\mathrm{C}-\mathrm{C})=0.003 \AA$ \\ $R$ factor $=0.059$ \\ $w R$ factor $=0.138$ \\ Data-to-parameter ratio $=15.3$
}

For details of how these key indicators were automatically derived from the article, see http://journals.iucr.org/e.

(C) 2006 International Union of Crystallography All rights reserved

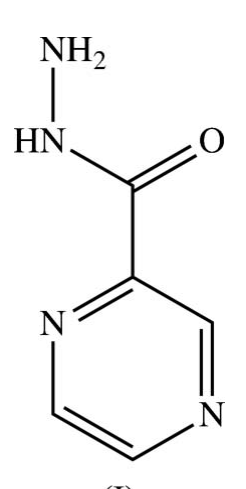

(I)

The molecules are linked by hydrogen bonds (Table 2) into a three-dimensional framework of some complexity, whose formation can, nonetheless, be readily analysed in terms of two simple substructures. In the first of these substructures, atom $\mathrm{N} 3$ in the molecule at $(x, y, z)$ acts as hydrogen-bond donor, via $\mathrm{H} 31$ and $\mathrm{H} 32$, respectively, to atoms $\mathrm{O} 1$ in the molecules at $(1-x, 1-y, 1-z)$ and $(-x, 1-y, 1-z)$, so generating by inversion a chain of edge-fused $R_{2}^{2}(10)$ (Bernstein et al., 1995) rings running along $\left(x, \frac{1}{2}, \frac{1}{2}\right)$ (Fig. 2). The rings containing $\mathrm{H} 31$ are centred at $\left(n+\frac{1}{2}, \frac{1}{2}, \frac{1}{2}\right)$, where $n=$ zero or an integer) and those containing $\mathrm{H} 32$ are centred at $\left(n, \frac{1}{2}, \frac{1}{2}\right)(n=$ zero or integer).

In the second substructure, atom $\mathrm{N} 2$ in the molecule at $(x, y$, $z)$, which lies in the chain of rings along $\left(x, \frac{1}{2}, \frac{1}{2}\right)$, acts as hydrogen-bond donor to atom N4 in the molecule at $(1-x$, $\left.\frac{1}{2}-y, \frac{1}{2}+z\right)$, which lies in the chain along $(x, 0,1)$; at the same time, atom C3 at $\left(1-x, \frac{1}{2}-y, \frac{1}{2}+z\right)$ acts as donor to atom N1 in the molecule at $(x, y, z)$, so forming an $R_{2}^{2}(8)$ motif (Fig. 3). Propagation of this motif by the symmetry operations of the space group then links the chain of rings along $\left(x, \frac{1}{2}, \frac{1}{2}\right)$ directly
Received 27 July 2006 Accepted 28 July 2006 


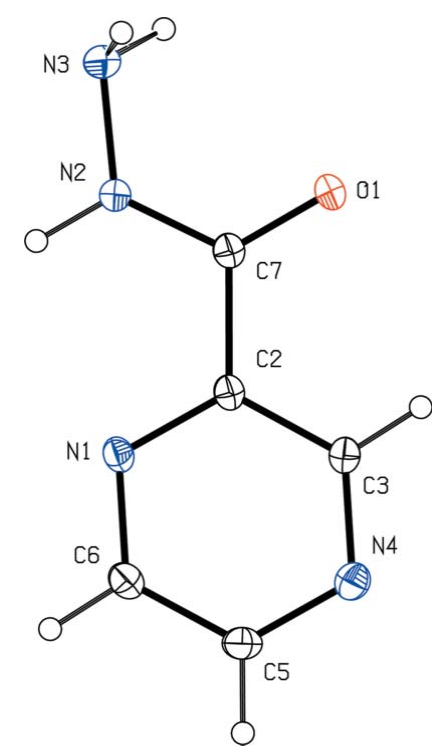

Figure 1

The molecular structure of compound (I), showing the atom-labelling scheme. Displacement ellipsoids are drawn at the $30 \%$ probability level.
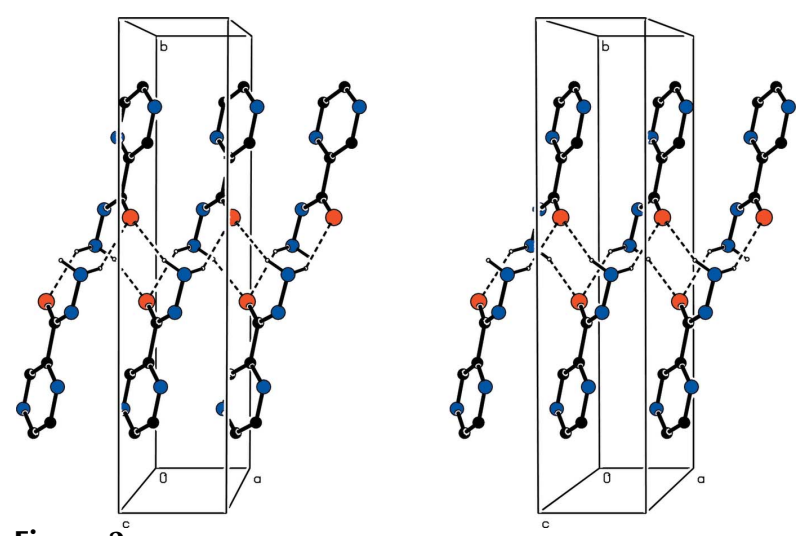

Figure $2^{\circ}$

A stereoview of part of the crystal structure of compound (I), showing the formation of a chain of edge-fused rings along $\left(x, \frac{1}{2}, \frac{1}{2}\right)$. For the sake of clarity, $\mathrm{H}$ atoms not involved in the motifs shown have been omitted.

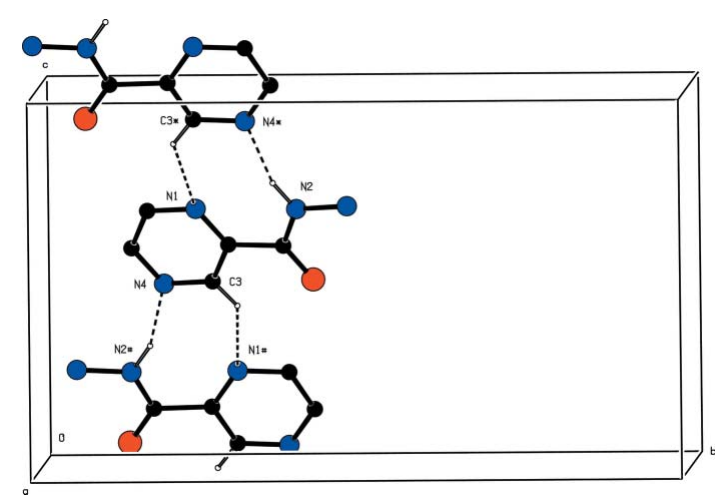

Figure 3

Part of the crystal structure of compound (I), showing the concerted action of the $\mathrm{N}-\mathrm{H} \cdots \mathrm{N}$ and $\mathrm{C}-\mathrm{H} \cdots \mathrm{N}$ hydrogen bonds. For the sake of clarity, $\mathrm{H}$ atoms not involved in the motif shown have been omitted. Atoms marked with an asterisk (*) or a hash (\#) are at the symmetry positions $\left(1+x, \frac{1}{2}-y, \frac{1}{2}+z\right)$ and $\left(-1+x, \frac{1}{2}-y,-\frac{1}{2}+z\right)$, respectively.

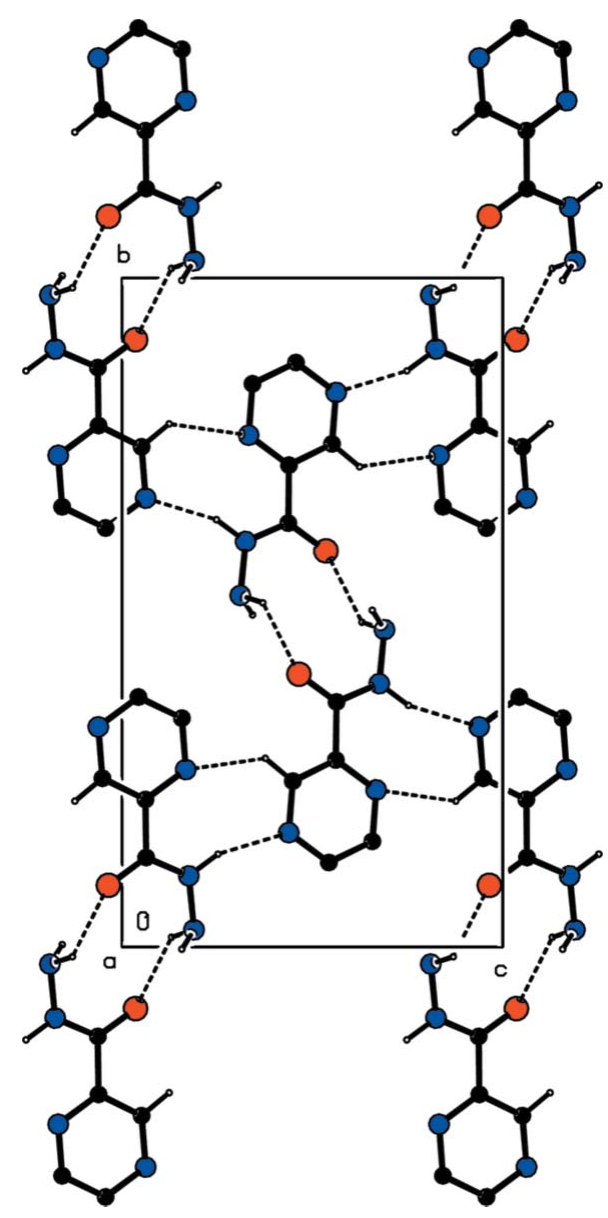

Figure 4

A projection down [100] of part of the crystal structure of compound (I), showing the linking of the chain of rings along $\left(x, \frac{1}{2}, \frac{1}{2}\right)$ to four adjacent chains. For the sake of clarity, $\mathrm{H}$ atoms not involved in the motifs shown have been omitted.

to the four chains along $(x, 0,0),(x, 0,1),(x, 1,0)$ and $(x, 1,1)$, thence linking all of the [100] chains into a single threedimensional framework structure (Fig. 4).

\section{Experimental}

A solution of methyl pyrazinecarboxylate and a fivefold molar excess of hydrazine hydrate was held at $353 \mathrm{~K}$ for $12 \mathrm{~h}$. The solvent was removed under reduced pressure and the residue was purified by washing successively with cold ethanol and with diethyl ether to give crystalline (I) (yield 87\%, m.p. 431-432 K). NMR (DMSO- $d_{6}$ ): $\delta(\mathrm{H})$ $10.14(1 \mathrm{H}, s, \mathrm{NH}), 9.13(1 \mathrm{H}, d, J=1.2 \mathrm{~Hz}, \mathrm{H} 3), 8.84(1 \mathrm{H}, d, J=2.8 \mathrm{~Hz}$, H6), $8.70(1 \mathrm{H}, d d, J=1.2$ and $2.8 \mathrm{~Hz}, \mathrm{H} 5), 4.70\left(2 \mathrm{H}, s, \mathrm{NH}_{2}\right) ; \delta(\mathrm{C})$ 161.4, 147.2, 144.8, 143.4, 143.1. IR (KBr disk, $\left.\mathrm{cm}^{-1}\right) 3306-3238(\mathrm{NH})$, $1648(\mathrm{CO})$.

\section{Crystal data}

$\mathrm{C}_{5} \mathrm{H}_{6} \mathrm{~N}_{4} \mathrm{O}$

$M_{r}=138.14$

Monoclinic, $P 2_{1} / c$

$a=3.7193(5) \AA$

$b=16.978(2) \AA$

$c=9.7858(10) \AA$

$\beta=99.185(8)$

$V=610.01(13) \AA^{3}$
$Z=4$

$D_{x}=1.504 \mathrm{Mg} \mathrm{m}^{-3}$

Mo $K \alpha$ radiation

$\mu=0.11 \mathrm{~mm}^{-1}$

$T=120$ (2) K

Plate, colourless

$0.50 \times 0.18 \times 0.01 \mathrm{~mm}$ 


\section{Data collection}

Bruker-Nonius KappaCCD diffractometer

$\varphi$ and $\omega$ scans

Absorption correction: multi-scan (SADABS; Sheldrick, 2003)

$T_{\min }=0.965, T_{\max }=0.999$

\section{Refinement}

Refinement on $F^{2}$

$R\left[F^{2}>2 \sigma\left(F^{2}\right)\right]=0.059$

$w R\left(F^{2}\right)=0.138$

$S=1.08$

1395 reflections

91 parameters

$\mathrm{H}$-atom parameters constrained

$$
\begin{gathered}
w=1 /\left[\sigma^{2}\left(F_{\mathrm{o}}{ }^{2}\right)+(0.0462 P)^{2}\right. \\
\quad+0.4947 P] \\
\text { where } P=\left(F_{\mathrm{o}}^{2}+2 F_{\mathrm{c}}{ }^{2}\right) / 3 \\
(\Delta / \sigma)_{\max }<0.001 \\
\Delta \rho_{\max }=0.20 \AA^{-3} \\
\Delta \rho_{\min }=-0.24 \mathrm{e}^{-3}
\end{gathered}
$$
1395 independent reflections $R_{\text {int }}=0.057$

$\theta_{\max }=27.5^{\circ}$

\section{Table 1}

Selected torsion angles $\left({ }^{\circ}\right)$.

\begin{tabular}{llll}
\hline $\mathrm{N} 1-\mathrm{C} 2-\mathrm{C} 7-\mathrm{N} 2$ & $-1.9(3)$ & $\mathrm{C} 2-\mathrm{C} 7-\mathrm{N} 2-\mathrm{N} 3$ & $179.60(18)$
\end{tabular}

\section{Table 2}

Hydrogen-bond geometry $\left(\AA,^{\circ}\right)$.

\begin{tabular}{lllll}
\hline$D-\mathrm{H} \cdots A$ & $D-\mathrm{H}$ & $\mathrm{H} \cdots A$ & $D \cdots A$ & $D-\mathrm{H} \cdots A$ \\
\hline $\mathrm{N} 2-\mathrm{H} 2 \cdots \mathrm{N} 44^{\mathrm{i}}$ & 0.96 & 2.06 & $2.976(3)$ & 160 \\
$\mathrm{~N} 3-\mathrm{H} 31 \cdots \mathrm{O} 1^{\text {ii }}$ & 0.92 & 2.31 & $3.079(3)$ & 140 \\
$\mathrm{~N} 3-\mathrm{H} 32 \cdots \mathrm{O} 1^{\mathrm{iii}}$ & 0.92 & 2.25 & $3.138(2)$ & 161 \\
$\mathrm{C} 3-\mathrm{H} 3 \cdots \mathrm{N} 1^{\text {iv }}$ & 0.95 & 2.59 & $3.312(3)$ & 133 \\
\hline
\end{tabular}

Symmetry codes: (i) $\quad x+1,-y+\frac{1}{2}, z+\frac{1}{2} ; \quad$ (ii) $\quad-x+1,-y+1,-z+1$; $-x,-y+1,-z+1$; (iv) $x-1,-y+\frac{1}{2}, z-\frac{1}{2}$.
All $\mathrm{H}$ atoms were located in difference maps, and then treated as riding atoms, with $\mathrm{C}-\mathrm{H}=0.95 \AA$ and $\mathrm{N}-\mathrm{H}=0.92\left(\mathrm{NH}_{2}\right)$ or $0.96 \AA$ $(\mathrm{NH})$, with $U_{\text {iso }}(\mathrm{H})=1.2 U_{\text {eq }}(\mathrm{C}, \mathrm{N})$.

Data collection: COLLECT (Hooft, 1999); cell refinement: $D E N Z O$ (Otwinowski \& Minor, 1997) and COLLECT; data reduction: DENZO and COLLECT; program(s) used to solve structure: OSCAIL (McArdle, 2003) and SHELXS97 (Sheldrick, 1997); program(s) used to refine structure: OSCAIL and SHELXL97 (Sheldrick, 1997); molecular graphics: PLATON (Spek, 2003); software used to prepare material for publication: SHELXL97 and PRPKAPPA (Ferguson, 1999).

X-Ray data were collected at the EPSRC National X-ray Crystallography Service, University of Southampton, England; the authors thank the staff of the Service for all their help and advice. JLW thanks CNPq and FAPERJ for financial support.

\section{References}

Bernstein, J., Davis, R. E., Shimoni, L. \& Chang, N.-L. (1995). Angew. Chem. Int. Ed. Engl. 34, 1555-1573.

Ferguson, G. (1999). PRPKAPPA. University of Guelph, Canada.

Hooft, R. W. W. (1999). COLLECT. Nonius BV, Delft, The Netherlands.

McArdle, P. (2003). OSCAIL for Windows. Version 10. Crystallography Centre, Chemistry Department, NUI Galway, Ireland.

Otwinowski, Z. \& Minor, W. (1997). Methods in Enzymology, Vol. 276, Macromolecular Crystallography, Part A, edited by C. W. Carter Jr \& R. M. Sweet, pp. 307-326. New York: Academic Press.

Sheldrick, G. M. (1997). SHELXS97 and SHELXL97. University of Göttingen, Germany.

Sheldrick, G. M. (2003). SADABS. Version 2.10. University of Göttingen, Germany.

Spek, A. L. (2003). J. Appl. Cryst. 36, 7-13. 


\section{supporting information}

Acta Cryst. (2006). E62, o3765-03767 [https://doi.org/10.1107/S1600536806029394]

Pyrazine-2-carbohydrazide: a three-dimensional hydrogen-bonded framework structure

Solange M. S. V. Wardell, Marcus V. N. de Souza, James L. Wardell, John N. Low and

\section{Christopher Glidewell}

Pyrazine-2-carbohydrazide

Crystal data

$\mathrm{C}_{5} \mathrm{H}_{6} \mathrm{~N}_{4} \mathrm{O}$

$M_{r}=138.14$

Monoclinic, $P 2_{1} / c$

Hall symbol: -P $2 \mathrm{ybc}$

$a=3.7193(5) \AA$

$b=16.978(2) \AA$

$c=9.7858(10) \AA$

$\beta=99.185(8)^{\circ}$

$V=610.01(13) \AA^{3}$

$Z=4$

$F(000)=288$

$D_{\mathrm{x}}=1.504 \mathrm{Mg} \mathrm{m}^{-3}$

Mo $K \alpha$ radiation, $\lambda=0.71073 \AA$

Cell parameters from 1395 reflections

$\theta=4.2-27.5^{\circ}$

$\mu=0.11 \mathrm{~mm}^{-1}$

$T=120 \mathrm{~K}$

Plate, colourless

$0.50 \times 0.18 \times 0.01 \mathrm{~mm}$

\section{Data collection}

Bruker-Nonius KappaCCD diffractometer

Radiation source: Bruker-Nonius FR591 rotating anode

Graphite monochromator

Detector resolution: 9.091 pixels $\mathrm{mm}^{-1}$

$\varphi$ and $\omega$ scans

Absorption correction: multi-scan

(SADABS; Sheldrick, 2003)

Refinement

Refinement on $F^{2}$

Least-squares matrix: full

$R\left[F^{2}>2 \sigma\left(F^{2}\right)\right]=0.059$

$w R\left(F^{2}\right)=0.138$

$S=1.08$

1395 reflections

91 parameters

0 restraints

Primary atom site location: structure-invariant direct methods
$T_{\min }=0.965, T_{\max }=0.999$

6568 measured reflections

1395 independent reflections

1080 reflections with $I>2 \sigma(I)$

$R_{\text {int }}=0.057$

$\theta_{\max }=27.5^{\circ}, \theta_{\min }=4.2^{\circ}$

$h=-4 \rightarrow 4$

$k=-22 \rightarrow 19$

$l=-12 \rightarrow 12$

Secondary atom site location: difference Fourier map

Hydrogen site location: inferred from neighbouring sites

$\mathrm{H}$-atom parameters constrained

$w=1 /\left[\sigma^{2}\left(F_{\mathrm{o}}^{2}\right)+(0.0462 P)^{2}+0.4947 P\right]$

where $P=\left(F_{\mathrm{o}}{ }^{2}+2 F_{\mathrm{c}}{ }^{2}\right) / 3$

$(\Delta / \sigma)_{\max }<0.001$

$\Delta \rho_{\max }=0.20 \mathrm{e} \AA^{-3}$

$\Delta \rho_{\min }=-0.24$ e $\AA^{-3}$ 
Fractional atomic coordinates and isotropic or equivalent isotropic displacement parameters $\left(\AA^{2}\right)$

\begin{tabular}{lllll}
\hline & $x$ & $y$ & $z$ & $U_{\text {iso }} * / U_{\text {eq }}$ \\
\hline N1 & $0.2754(5)$ & $0.23490(10)$ & $0.66747(17)$ & $0.0223(4)$ \\
C2 & $0.1216(6)$ & $0.27960(12)$ & $0.5616(2)$ & $0.0199(5)$ \\
C3 & $-0.1032(6)$ & $0.24786(13)$ & $0.4486(2)$ & $0.0218(5)$ \\
N4 & $-0.1773(5)$ & $0.17092(11)$ & $0.43778(19)$ & $0.0255(4)$ \\
C5 & $-0.0225(6)$ & $0.12634(13)$ & $0.5431(2)$ & $0.0269(5)$ \\
C6 & $0.1998(6)$ & $0.15799(13)$ & $0.6573(2)$ & $0.0255(5)$ \\
C7 & $0.1993(6)$ & $0.36628(12)$ & $0.5634(2)$ & $0.0214(5)$ \\
O1 & $0.0679(4)$ & $0.40779(9)$ & $0.46448(15)$ & $0.0273(4)$ \\
N2 & $0.4142(5)$ & $0.39368(10)$ & $0.67541(18)$ & $0.0237(4)$ \\
N3 & $0.5100(6)$ & $0.47441(11)$ & $0.6892(2)$ & $0.0305(5)$ \\
H2 & 0.5180 & 0.3612 & 0.7520 & $0.028^{*}$ \\
H3 & -0.2086 & 0.2820 & 0.3763 & $0.026^{*}$ \\
H5 & -0.0659 & 0.0712 & 0.5400 & $0.032^{*}$ \\
H6 & 0.3014 & 0.1239 & 0.7304 & $0.031^{*}$ \\
H31 & 0.6722 & 0.4855 & 0.6300 & $0.037^{*}$ \\
H32 & 0.3048 & 0.5034 & 0.6571 & $0.037^{*}$
\end{tabular}

Atomic displacement parameters $\left(\AA^{2}\right)$

\begin{tabular}{lllllll}
\hline & $U^{11}$ & $U^{22}$ & $U^{33}$ & $U^{12}$ & $U^{13}$ & $U^{23}$ \\
\hline $\mathrm{N} 1$ & $0.0249(10)$ & $0.0235(10)$ & $0.0180(8)$ & $0.0030(7)$ & $0.0020(7)$ & $0.0022(7)$ \\
$\mathrm{C} 2$ & $0.0195(10)$ & $0.0232(11)$ & $0.0173(10)$ & $0.0034(8)$ & $0.0036(8)$ & $0.0002(8)$ \\
$\mathrm{C} 3$ & $0.0207(10)$ & $0.0253(11)$ & $0.0189(10)$ & $0.0014(9)$ & $0.0018(8)$ & $-0.0008(8)$ \\
$\mathrm{N} 4$ & $0.0252(10)$ & $0.0275(11)$ & $0.0238(9)$ & $-0.0001(8)$ & $0.0038(8)$ & $-0.0027(7)$ \\
$\mathrm{C} 5$ & $0.0317(13)$ & $0.0203(11)$ & $0.0291(12)$ & $-0.0021(9)$ & $0.0067(10)$ & $-0.0001(9)$ \\
$\mathrm{C} 6$ & $0.0284(12)$ & $0.0242(11)$ & $0.0239(11)$ & $0.0042(9)$ & $0.0046(9)$ & $0.0031(9)$ \\
$\mathrm{C} 7$ & $0.0211(11)$ & $0.0231(11)$ & $0.0200(10)$ & $0.0020(9)$ & $0.0028(8)$ & $0.0008(8)$ \\
$\mathrm{O} 1$ & $0.0316(9)$ & $0.0237(8)$ & $0.0236(8)$ & $0.0014(7)$ & $-0.0048(6)$ & $0.0042(6)$ \\
$\mathrm{N} 2$ & $0.0279(10)$ & $0.0201(10)$ & $0.0213(9)$ & $-0.0011(7)$ & $-0.0017(7)$ & $0.0006(7)$ \\
$\mathrm{N} 3$ & $0.0340(11)$ & $0.0210(10)$ & $0.0333(11)$ & $-0.0015(8)$ & $-0.0039(8)$ & $0.0001(8)$ \\
& & & & & & \\
\hline
\end{tabular}

Geometric parameters $\left(\AA,{ }^{\circ}\right)$

\begin{tabular}{llll}
\hline $\mathrm{N} 1-\mathrm{C} 6$ & $1.336(3)$ & $\mathrm{N} 3-\mathrm{H} 31$ & 0.92 \\
$\mathrm{~N} 1-\mathrm{C} 2$ & $1.337(3)$ & $\mathrm{N} 3-\mathrm{H} 32$ & 0.92 \\
$\mathrm{C} 2-\mathrm{C} 3$ & $1.385(3)$ & $\mathrm{C} 3-\mathrm{N} 4$ & 0.95 \\
$\mathrm{C} 2-\mathrm{C} 7$ & $1.499(3)$ & $\mathrm{C} 3-\mathrm{H} 3$ & $1.333(3)$ \\
$\mathrm{C} 7-\mathrm{O} 1$ & $1.234(2)$ & $\mathrm{N} 4-\mathrm{C} 5$ & $1.388(3)$ \\
$\mathrm{C} 7-\mathrm{N} 2$ & $1.333(3)$ & $\mathrm{C} 5-\mathrm{C} 6$ & 0.95 \\
$\mathrm{~N} 2-\mathrm{N} 3$ & $1.417(3)$ & $\mathrm{C} 5-\mathrm{H} 5$ & 0.95 \\
$\mathrm{~N} 2-\mathrm{H} 2$ & 0.96 & $\mathrm{C} 6-\mathrm{H} 6$ & 105.5 \\
$\mathrm{C} 6-\mathrm{N} 1-\mathrm{C} 2$ & & $\mathrm{H} 31-\mathrm{N} 3-\mathrm{H} 32$ & $122.32(19)$ \\
$\mathrm{N} 1-\mathrm{C} 2-\mathrm{C} 3$ & $115.99(18)$ & $\mathrm{N} 4-\mathrm{C} 3-\mathrm{C} 2$ & 118.8 \\
$\mathrm{~N} 1-\mathrm{C} 2-\mathrm{C} 7$ & $121.8(2)$ & $\mathrm{N} 4-\mathrm{C} 3-\mathrm{H} 3$ &
\end{tabular}




\begin{tabular}{|c|c|c|c|c|}
\hline $\mathrm{C} 3-\mathrm{C} 2-\mathrm{C} 7$ & $118.81(18)$ & $\mathrm{C} 2-\mathrm{C} 3-\mathrm{H} 3$ & & 118.8 \\
\hline $\mathrm{O} 1-\mathrm{C} 7-\mathrm{N} 2$ & $123.8(2)$ & $\mathrm{C} 5-\mathrm{N} 4-\mathrm{C} 3$ & & $115.82(19)$ \\
\hline $\mathrm{O} 1-\mathrm{C} 7-\mathrm{C} 2$ & $120.01(18)$ & $\mathrm{N} 4-\mathrm{C} 5-\mathrm{C} 6$ & & $122.1(2)$ \\
\hline $\mathrm{N} 2-\mathrm{C} 7-\mathrm{C} 2$ & $116.19(17)$ & $\mathrm{N} 4-\mathrm{C} 5-\mathrm{H} 5$ & & 119.0 \\
\hline $\mathrm{C} 7-\mathrm{N} 2-\mathrm{N} 3$ & $121.63(18)$ & $\mathrm{C} 6-\mathrm{C} 5-\mathrm{H} 5$ & & 119.0 \\
\hline $\mathrm{C} 7-\mathrm{N} 2-\mathrm{H} 2$ & 123.7 & $\mathrm{~N} 1-\mathrm{C} 6-\mathrm{C} 5$ & & $122.0(2)$ \\
\hline $\mathrm{N} 3-\mathrm{N} 2-\mathrm{H} 2$ & 114.6 & $\mathrm{~N} 1-\mathrm{C} 6-\mathrm{H} 6$ & & 119.0 \\
\hline $\mathrm{N} 2-\mathrm{N} 3-\mathrm{H} 31$ & 108.5 & $\mathrm{C} 5-\mathrm{C} 6-\mathrm{H} 6$ & & 119.0 \\
\hline $\mathrm{N} 2-\mathrm{N} 3-\mathrm{H} 32$ & 107.6 & & & \\
\hline $\mathrm{C} 6-\mathrm{N} 1-\mathrm{C} 2-\mathrm{C} 3$ & $0.2(3)$ & $\mathrm{C} 2-\mathrm{C} 7-\mathrm{N} 2-\mathrm{N} 3$ & & $179.60(18)$ \\
\hline $\mathrm{C} 6-\mathrm{N} 1-\mathrm{C} 2-\mathrm{C} 7$ & $-178.60(18)$ & $\mathrm{N} 1-\mathrm{C} 2-\mathrm{C} 3-\mathrm{N} 4$ & & $-0.7(3)$ \\
\hline $\mathrm{N} 1-\mathrm{C} 2-\mathrm{C} 7-\mathrm{O} 1$ & $177.82(19)$ & $\mathrm{C} 7-\mathrm{C} 2-\mathrm{C} 3-\mathrm{N} 4$ & & $178.14(19)$ \\
\hline $\mathrm{C} 3-\mathrm{C} 2-\mathrm{C} 7-\mathrm{O} 1$ & $-1.0(3)$ & $\mathrm{C} 2-\mathrm{C} 3-\mathrm{N} 4-\mathrm{C} 5$ & & $0.4(3)$ \\
\hline $\mathrm{N} 1-\mathrm{C} 2-\mathrm{C} 7-\mathrm{N} 2$ & $-1.9(3)$ & $\mathrm{C} 3-\mathrm{N} 4-\mathrm{C} 5-\mathrm{C} 6$ & & $0.4(3)$ \\
\hline $\mathrm{C} 3-\mathrm{C} 2-\mathrm{C} 7-\mathrm{N} 2$ & $179.31(18)$ & $\mathrm{C} 2-\mathrm{N} 1-\mathrm{C} 6-\mathrm{C} 5$ & & $0.5(3)$ \\
\hline $\mathrm{O} 1-\mathrm{C} 7-\mathrm{N} 2-\mathrm{N} 3$ & $-0.1(3)$ & $\mathrm{N} 4-\mathrm{C} 5-\mathrm{C} 6-\mathrm{N} 1$ & & $-0.8(3)$ \\
\hline \multicolumn{5}{|c|}{ Hydrogen-bond geometry $\left(\hat{A},{ }^{o}\right)$} \\
\hline$D-\mathrm{H} \cdots A$ & $D-\mathrm{H}$ & $\mathrm{H} \cdots A$ & $D \cdots A$ & $D-\mathrm{H} \cdots A$ \\
\hline $\mathrm{N} 2-\mathrm{H} 2 \cdots \mathrm{N} 4^{\mathrm{i}}$ & 0.96 & 2.06 & $2.976(3)$ & 160 \\
\hline $\mathrm{N} 3-\mathrm{H} 31 \cdots \mathrm{O} 1^{\mathrm{ii}}$ & 0.92 & 2.31 & $3.079(3)$ & 140 \\
\hline $\mathrm{N} 3-\mathrm{H} 32 \cdots \mathrm{O} 1^{\mathrm{iii}}$ & 0.92 & 2.25 & $3.138(2)$ & 161 \\
\hline $\mathrm{C} 3-\mathrm{H} 3 \cdots \mathrm{N} 1^{\mathrm{iv}}$ & 0.95 & 2.59 & $3.312(3)$ & 133 \\
\hline
\end{tabular}

Symmetry codes: (i) $x+1,-y+1 / 2, z+1 / 2$; (ii) $-x+1,-y+1,-z+1$; (iii) $-x,-y+1,-z+1$; (iv) $x-1,-y+1 / 2, z-1 / 2$. 